\title{
Cardiopulmonary resuscitation - time for a change in the paradigm?
}

Michele Levinson MD, FRACP, FCICM Head of Department

Amber Mills PhD, BA(Hons) Research Fellow

Cabrini-Monash University Department of Medicine, Monash University, Melbourne, VIC.

mlevinson@ cabrini.com.au

doi: $10.5694 / \mathrm{mial} 4.00012$

do: $10.5694 / \mathrm{mja} 4.00012$

.

ince the description and implementation of cardiopulmonary resuscitation (CPR) as a lifesaving procedure in the 1960s, it has become the default treatment for all patients dying in hospitals around Australia unless documentation of a not-for-resuscitation (NFR) plan or order is recorded in the patient's file. At the same time, the number of elderly patients with geriatric syndromes admitted to Australian hospitals has increased. Use of CPR and NFR orders is of particular relevance to this group as they have poor survival and outcomes following resuscitation. ${ }^{1}$ The aim of this paper is to question whether all patients should receive CPR as a default treatment.

NFR documentation can take many forms, from a handwritten comment by a doctor in the patient's file to a form specifically designed for the purpose. The procedure is also referred to as do not resuscitate (DNR) and do not attempt to resuscitate (DNAR). For the purposes of this article, it will be referred to as NFR. Most NFR forms include a section that records whether the NFR decision has been discussed with the patient or the patient's family. In some cases there is a need to declare whether the patient is competent to make a decision about CPR. Australian data on the prevalence of NFR forms in inpatient histories show that only a minority of older general medical patients have NFR documented in their history. ${ }^{2}$

Survival to discharge after CPR in the chronically ill older hospital population is poor - less than $6 \%$. Discharge home (as a post-CPR outcome) has decreased without a change in overall survival. ${ }^{3}$ A significant proportion of those who survive to hospital discharge die within the first postdischarge month. Orotracheal intubation during cardiac arrest, age, premorbid residence in a nursing facility and more comorbid illness predict a poor outcome from CPR. . $^{4-6}$

Several studies have shown that up to one-quarter of all medical emergency team (MET) calls result in a new allocation of NFR status or an assessment that this would be appropriate. In $90 \%$ of cases there was agreement between the primary care clinician and the MET assessment of suggested NFR status. In an Australian study, patients who had a limitation of medical treatment instituted by the MET had a hospital mortality of 52\%. ${ }^{7,8}$ NFR orders are implemented only for a minority of hospital patients who are unlikely to benefit from CPR. ${ }^{2}$ When NFR orders are written for general medical inhospital patients, they tend to be written for those already recognised as dying or at high immediate risk of death. NFR orders seem to be functioning as an indicator of imminent death. A lack of NFR orders may preclude attention to appropriate end-of-life care ${ }^{9}$ and exposes the patient to a potentially brutal unsuccessful intervention that is highly

Online first 28/07/14

\section{Sunnาary}

- Cardiopulmonary resuscitation (CPR) is the default treatment in hospital unless there is a decision to the contrary and this is documented in the patient record.

- The outcome of CPR in older chronically ill patients is very poor and discharge home is unlikely.

- Fewer not-for-resuscitation (NFR) orders are written than there are patients who would not benefit from CPR.

- NFR orders appear to be a marker of death, rather than the result of informed discussion about end-of-life care.

There is a legal and ethical framework for the consideration of the suitability of CPR.

- Discussions about CPR are challenging, and uncertainty is introduced because of the lack of consensus around futility, the emotionally charged nature of the topic, misconceptions about the success of CPR and the failure to recognise that not offering CPR will allow a peaceful and supported death.

- Discussion around CPR can be misconstrued as a need for consent. A focus on patient and family involvement may result in an expectation that CPR is an entitlement.

- As part of evidence-based patient-centred care, CPR should only be offered to those for whom it is beneficial.

- CPR should no longer be the universal default. We propose an opt-in model, which will drive discussion and evaluation of the efficacy and suitability of CPR for the individual.

A CPR discussion should occur on admission for all elderly hospital inpatients.

There is a legal and ethical framework for consideration of CPR based on futility, non-maleficence, beneficence, autonomy and the law.

\section{Futility}

A medical professional is under no obligation to provide treatment where no benefit at all would be conferred; however, the meanings of benefit and futility are highly contested. Willmott and colleagues state that the meanings of futile and futility are uncertain in law, and that greater clarification is required. ${ }^{11}$ They define treatment as futile when there is medical consensus to this effect. Although there is often consensus among the treating doctors that an older chronically ill patient is unlikely to survive CPR, the decision not to offer CPR on the grounds of futility could still be legally challenged. There is no standard Australian guidance on how to achieve consensus, who should participate in it or how consensus should be documented. In the event of dispute about futility, those close to the patient may test 
1 Circumstances where futility considerations are relevant

advanced dementia; particularly in association with low body weight

New York Heart Association Class IV heart failure

end-stage renal failure where dialysis is not an option

advanced frailty

recurrent aspiration

large stroke with poor functional recovery

end-stage chronic obstructive pulmonary disease

advanced malignancy refractory to treatment

the medical practitioner's determination of futility in the Supreme Court. ${ }^{12}$ This is likely to undermine the medical practitioner's confidence in invoking futility as a reason to withhold CPR. Similarly, the Canadian Joint statement on resuscitative interventions (update 1995) does not define futility but does encourage involvement of the patient in determining futility, although lack of competence or delirium will hamper participation. ${ }^{13}$

The presence of poor prognostic factors may be sufficient to consider CPR futile before cardiorespiratory arrest occurs. We have included a list of some circumstances where a judgement of futility may be made (Box 1). In other instances, futility can only be ascertained at the time of cardiac arrest, through relevant factors such as asystole and pulseless electrical activity. ${ }^{5,6}$ Regardless, we argue that physicians be encouraged and supported to make a deliberate decision about resuscitation, rather than rely on the default approach.

\section{Non-maleficence}

A medical professional is under obligation to ensure they do no harm, and this often entails a balance between benefit and futility of treatment. Hayes suggests that the decision to withhold CPR in the medically unwell but not imminently dying patient is a judgement about providing an unsuccessful treatment which incurs harm by denying the patient a peaceful death. ${ }^{9}$

Following a technical medical judgement that CPR would not benefit and may harm in this situation, a CPR-related "deliberative discussion" with the patient and family is important in order to prevent harmful misunderstanding, as they may expect CPR to be performed. If the patient or family disagree with the health care team, there needs to be an ethical decision made weighing up the harm to the patient by providing CPR with the harm to the trust between the

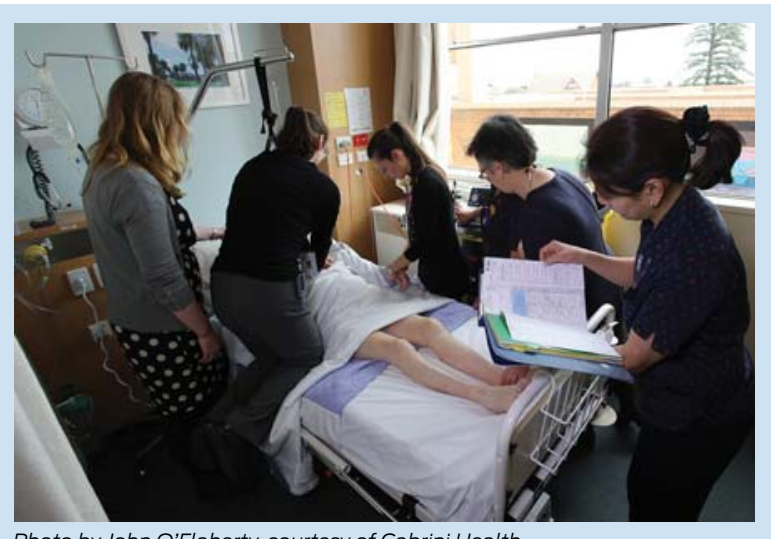

patient and family and the health care provider by not providing CPR. The loss of trust may compromise ongoing care. In the case where the patient is expected to survive CPR but have a poor outcome or for whom the outcome is uncertain, Hayes recommends an "interpretive discussion" about the value of CPR for the individual patient. The efficacy of these discussions can be seen in the improved end-of-life care and outcomes for patients and their families. ${ }^{14}$

Sources of disagreement between the patient and family and the health care provider may relate to lack of trust in health care staff; an overestimation of the success of CPR in the community, likely informed by the media portrayal of CPR; and the influence of community education which emphasises the provision of CPR as first aid., ${ }^{9,15}$ Religious and cultural beliefs that regard the sanctity of human life as paramount are likely to drive a CPR decision if there is any perceived potential for a deathbed miracle. However, many other treatment options within the practice of medicine are not offered to patients on the grounds that the treatment would not confer benefit or incur harm.

In the case of the sick, older frail patient, not providing CPR can be considered fulfilling the principles of beneficence and non-maleficence.

\section{Consent and autonomy}

Doctors have a professional, ethical and legal obligation to enable a patient to provide informed consent to or refusal of treatment. A patient's consent may be regarded as informed if it is voluntary and made with knowledge and understanding of the material benefits and risks involved. ${ }^{16}$ Notification to the Medical Board may result from failure to meet the requisite duty of disclosure. A breach of this duty causing harm may expose the doctor to liability in negligence.

It is clearly not possible to obtain consent from the patient for CPR at the time of need as by definition the patient is dead or near dead. CPR is provided on the understanding that it is an emergency procedure. However, patient autonomy can be preserved using advance care directives and communication with medical staff. ${ }^{12}$ Older patients are less likely to choose a burdensome treatment such as CPR if they understand that the probability of survival is low and if the outcome is poor functional outcome or cognitive impairment. ${ }^{17-19}$

The situation involving a cognitively impaired or demented older adult is more complex. Under Victorian law, and each state differs, only a guardian with the power to make medical decisions or person holding an enduring power of attorney (medical) can make a decision on behalf of the incompetent patient to withhold treatment. ${ }^{20}$ Patient-centred health care encourages the involvement of the patient and family in health care decisions. Discussions about hypothetical imminent death are highly charged, and patients may find the discussion about CPR challenging and feel that they are not able to engage in it; or alternatively, may strongly wish to engage. Heyland and colleagues showed that seriously ill patients and families do not want inappropriate life-prolonging measures, and that most wanted a shared decision-making role in discussions of end-of-life care. ${ }^{17}$ However, families often confuse the NFR discussion with consent seeking rather than information sharing. This is confounded further when the treating doctor is uninformed of the legal issues and abdicates responsibility for the decision to the family. ${ }^{21}$ 


\section{Case vignette}

An 86-year-old frail woman with advanced dementia and both urinary and faecal incontinence is admitted from a high-level care residential facility with septic shock secondary to infection with a highly resistant gram-negative organism. Treatment is commenced in the emergency department with intravenous antibiotics and fluids. The consultant physician (CP) responsible for the patient believes a resuscitation attempt would be futile if the patient continues to decline to death despite treatment. The CP discusses a not-for-resuscitation (NFR) order with the patient's daughter as is indicated in the hospital's protocol. The CP explains that cardiopulmonary resuscitation (CPR) would not be offered because of the lack of benefit that CPR would have in preventing her mother's death. It was explained that this was based on her mother's significant premorbid frailty and current serious illness.

The daughter reluctantly agrees with the CP's opinion. The following day the patient's daughter confronts the CP in the ward and demands that the NFR documentation be withdrawn. Consensus is not able to be achieved despite further discussion. The CP elects to cancel the NFR documentation rather than escalate the issue to medical administration.

The patient recovers and is discharged back to the high-level care residential facility.

\section{The way forward}

There are legal, social, administrative and knowledge barriers to "de-prescribing" CPR. We need open community discussion about end-of-life care. The discussion should demystify death and raise the situation-related efficacy of $\mathrm{CPR}$ and the importance of available community supports for death with dignity. Legal clarity is required for the concept of futility. Although medical and nursing professional bodies endorse the legal position of treatment consent and refusal and the concept of burdensome care in their published documents, the concepts are not yet part of the wider community conversation. Leadership from senior clinicians and legal counsel is needed to facilitate open discourse. Patient-centred evidence-based care demands that the role of CPR in Australian hospitals is reviewed. We believe that CPR should no longer be considered the universal default for all patients, and encourage debate.

Acknowledgements: We acknowledge and thank Bebe Loff (Director, Michael Kirby Centre for Public Health and Human Rights, Monash University) for her comments and assistance with the draft manuscript.

Competing interests: No relevant disclosures.

Provenance: Not commissioned; externally peer reviewed.

1 Paniagua D, Lopez-Jimenez F, Londoño JC, et al. Outcome and cost-effectiveness of cardiopulmonary resuscitation after in-hospital cardiac arrest in octogenarians. Cardiology 2002; 97: 6-11.

2 Shanmuganathan N, Li JY, Yong TY, et al. Resuscitation orders and their relevance to patients' clinical status and outcomes. QJM 2011; 104: 485-488.

3 Ehlenbach WJ, Barnato AE, Curtis JR, et al. Epidemiologic study of in-hospital cardiopulmonary resuscitation in the elderly. NEngl J Med 2009; 361: 22-31.

4 Cooper S, Janghorbani M, Cooper G. A decade of in-hospital resuscitation: outcomes and prediction of survival? Resuscitation 2006; 68: 231-237.

5 Weil MH, Fries M. In-hospital cardiac arrest. Crit Care Med 2005; 33: 2825-2830.

6 Di Bari M, Chiarlone M, Fumagalli S, et al. Cardiopulmonary resuscitation of older, inhospital patients: immediate efficacy and long-term outcome. Crit Care Med 2000; 28: 2320-2325.

7 Jones DA, Bagshaw SM, Barrett J, et al. The role of the medical emergency team in end-of-life care: a multicenter, prospective, observational study. Crit Care Med 2012; 40: 98-103.

8 Micallef S, Skrifvars MB, Parr MJ. Level of agreement on resuscitation decisions among hospital specialists and barriers to documenting do not attempt resuscitation (DNAR) orders in ward patients. Resuscitation 2011; 82: 815-818.

9 Hayes B. Clinical model for ethical cardiopulmonary resuscitation decision-making. Intern Med J 2013; 43 . 77-83.

10 Wallace CK. In-hospital cardiopulmonary resuscitation [letter]. N Engl J Med 2009; 361: 1708.

11 Willmott L, White B, Parker M, Cartwright C. The legal role of medical professionals in decisions to withhold or withdraw life-sustaining treatment: Part 3 (Victoria). J Law Med 2011; 18: 773-797.

12 Stewart CL. Legal perspectives on consent in disputes about futile care. Med J Aust 2013; 198: 225-226.

13 Joint statement on resuscitative interventions (update 1995). CMA policy summary. CMAJ 1995; 153: $1652 A-1652 \mathrm{~F}$.

14 Walling AM, Asch SM, Lorenz KA, et al. The quality of care provided to hospitalized patients at the end of life. Arch Intern Med 2010; 170: 1057-1063.

15 Jones GK, Brewer KL, Garrison HG. Public expectations of survival following cardiopulmnary resuscitation. Acad Emerg Med 2000; 7: 48-53.

16 Australian Medical Council. Good medical practice: a code of conduct for doctors in Australia. www.amc.org au/index.php/about/good-medical-practice (accessed Apr 2014).

17 Heyland DK, Dodek P, Rocker G, et al. What matters most in end-of-life care: perceptions of seriously ill patients and their family members. CMAJ 2006; 174: 627-633.

18 Murphy DJ, Burrows D, Santilli S, et al. The influence of the probability of survival on patients' preferences regarding cardiopulmonary resuscitation. N Engl J Med 1994; 330: 545-549.

19 Fried TR, Bradley EH, Towle VR, Allore H. Understanding the treatment preferences of seriously ill patients. NEngl J Med 2002; 346: 1061-1066.

20 Office of the Public Advocate. Advocacy and decision-making in relation to medical and dental treatment and other health care. http://www.publicadvocate.vic.gov.au/file/file/PracticeGuidelines/medical_ practice_guideline_December\%202010.pdf (accessed Apr 2014).

21 Murphy BF. What has happened to clinical leadership in futile care discussions? Med J Aust 2008; 188 : 418-419.

22 Le BH, Chapman MD. Competence and capacity at the end of life: uneasy paternalism. Med J Aust 2011; 195: 476-477.

23 Brauner DJ, Grusin SL. In-hospital cardiopulmonary resuscitation. N Engl J Med 2009; 361: 1708.

24 Mitchell SL, Black BS, Ersek M, et al. Advanced dementia: state of the art and priorities for the next decade. Ann Intern Med 2012; 156 (1 Pt 1): 45-51.

25 Ehlenbach WJ, Curtis JR. In-hospital cardiopulmonary resuscitation. N Engl J Med 2009; 361: 1708-1709. 